\title{
Multicritical behavior of two-dimensional anisotropic antiferromagnets in a magnetic field.
}

\author{
Andrea Pelissetto ${ }^{1}$ and Ettore Vicari $^{2}$ \\ ${ }^{1}$ Dipartimento di Fisica dell'Università di Roma "La Sapienza" and INFN, Roma, Italy. \\ 2 Dipartimento di Fisica dell'Università di Pisa and INFN, Pisa, Italy.
}

(Dated: March 25, 2022)

\begin{abstract}
We study the phase diagram and multicritical behavior of anisotropic Heisenberg antiferromagnets on a square lattice in the presence of a magnetic field along the easy axis. We argue that, beside the Ising and XY critical lines, the phase diagram presents a first-order spin-flop line starting from $T=0$, as in the three-dimensional case. By using field-theory methods, we show that the multicritical point where these transition lines meet cannot be $\mathrm{O}(3)$ symmetric and occurs at finite temperature. We also predict how the critical temperature of the transition lines varies with the magnetic field and the uniaxial anisotropy in the limit of weak anisotropy.
\end{abstract}

PACS numbers: $64.60 . \mathrm{Kw}, 05.10 . \mathrm{Cc}, 05.70 . J \mathrm{k}, 75.10 . \mathrm{Hk}$ 


\section{INTRODUCTION}

Anisotropic antiferromagnets in an external magnetic field have been studied for a long time. In order to determine their phase diagram, they have often been modelled by using the classical XXZ model

$$
\mathcal{H}=J \sum_{\langle m n\rangle} \vec{S}_{n} \cdot \vec{S}_{m}+A \sum_{\langle m n\rangle} S_{m, z} S_{n, z}-H \sum_{m} S_{m, z}
$$

where $\langle m n\rangle$ indicates a nearest-neighbor pair. Equivalently, one can use the Hamiltonian

$$
\mathcal{H}=J \sum_{\langle m n\rangle} \vec{S}_{n} \cdot \vec{S}_{m}+D \sum_{m} S_{m, z}^{2}-H \sum_{m} S_{m, z},
$$

with a single-ion anisotropy term. The most interesting case corresponds to uniaxial systems that show a complex phase diagram. They correspond to Hamiltonians with $A>0$ or $D<0$. Several quasi-two-dimensional uniaxial antiferromagnets have been studied experimentally, such as $\mathrm{K}_{2} \mathrm{MnF}_{4}, \mathrm{Rb}_{2} \mathrm{MnF}_{4}, \mathrm{Rb}_{2} \mathrm{MnCl}_{4} \cdot{ }^{1,2,3,4,5,6,7}$

Some general features of the phase diagram of anisotropic antiferromagnets are well known. Their phase diagram in the $T-H$ plane presents two critical lines, belonging to the Ising and XY universality class, respectively, which meet at a multicritical point (MCP). The nature of the MCP has been the object of several theoretical studies. In three dimensions (3D), the issue has been recently studied using a field-theory approach. ${ }^{8,9}$ The starting point is the $\mathrm{O}\left(n_{1}\right) \oplus \mathrm{O}\left(n_{2}\right)$ symmetric Landau-Ginzburg-Wilson (LGW) $\Phi^{4}$ theory $^{10}$

$$
\begin{aligned}
\mathcal{H}_{\mathrm{LGW}}= & \int d^{d} x\left\{\frac{1}{2}\left[\left(\partial_{\mu} \Phi_{1}\right)^{2}+\left(\partial_{\mu} \Phi_{2}\right)^{2}\right]+\frac{1}{2}\left[r_{1} \Phi_{1}^{2}+r_{2} \Phi_{2}^{2}\right]\right. \\
& \left.+\frac{1}{4 !}\left[u_{10}\left(\Phi_{1}^{2}\right)^{2}+u_{20}\left(\Phi_{2}^{2}\right)^{2}\right]+\frac{1}{4} w_{0} \Phi_{1}^{2} \Phi_{2}^{2}\right\}
\end{aligned}
$$

where $\Phi_{1}$ and $\Phi_{2}$ are vector fields with $n_{1}$ and $n_{2}$ real components, respectively. In our case $n_{1}=2$ and $n_{2}=1$ so that Hamiltonian (3) is symmetric under $\mathbb{Z}_{2} \oplus \mathrm{O}(2)$ transformations. The RG flow has been studied by computing and analyzing high-order perturbative expansions, ${ }^{8,9}$ to five and six loops. It has been shown that the stable fixed point (FP) of the theory is the biconal FP, and that no enlargement of the symmetry to $\mathrm{O}(3)$ must be asymptotically expected because the corresponding $\mathrm{O}(3) \mathrm{FP}$ is unstable, correcting earlier claims ${ }^{10}$ based on low-order $\epsilon$-expansion calculations. The perturbative results allow us to predict that the transition at the MCP is either continuous and belongs to the biconal universality class or that it is of first order - this occurs if the system is not in the attraction 


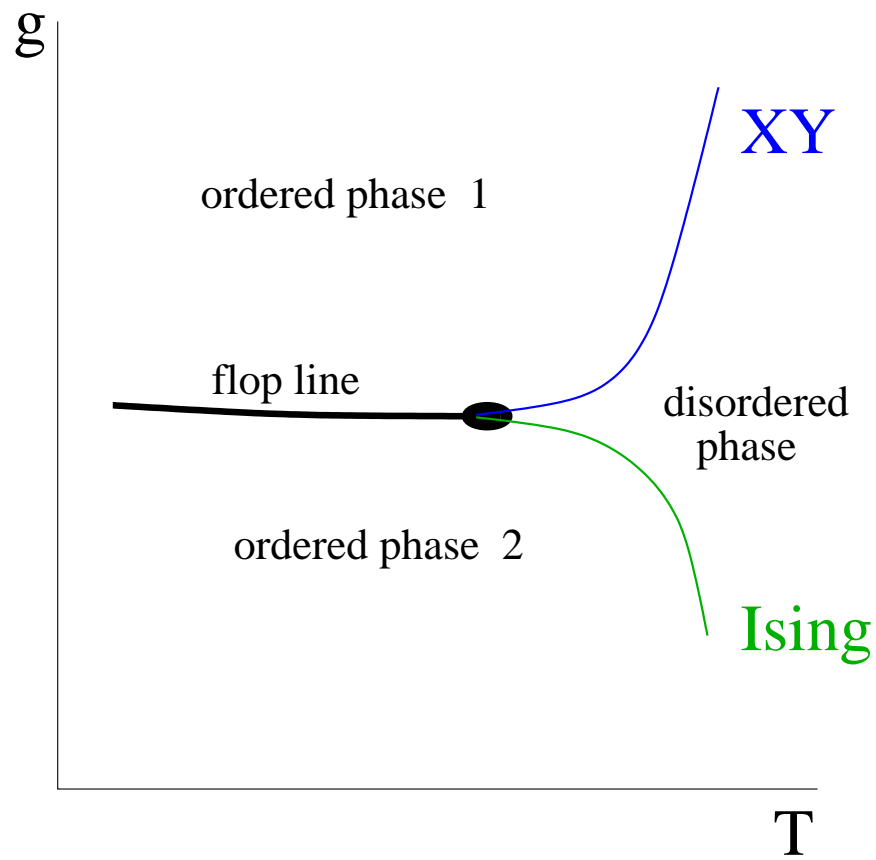

FIG. 1: Phase diagram with a bicritical point where an Ising and an XY line meet. Here, $T$ is the temperature and $g$ a second relevant parameter. The thick black line ("flop line") corresponds to a first-order transition.

domain of the stable biconal FP. Which of these two possibilities occurs is still an open issue. A mean-field analysis ${ }^{10,11}$ shows that the MCP is bicritical if $\delta_{0} \equiv u_{10} u_{20}-9 w_{0}^{2}<0$, and tetracritical if $\delta_{0}>0$. Figs. 1 and 2 sketch the corresponding phase diagrams. At one loop in the $\epsilon$ expansion the biconal FP is associated with a tetracritical phase diagram. ${ }^{10}$ Since uniaxial antiferromagnets have a bicritical phase diagram, this result predicts a first-order MCP as in Fig. 3. First-order transitions are also expected along the critical lines that separate the disordered phase from the ordered ones. They start at the MCP, extend up to tricritical points, and are followed by lines of XY and Ising transitions.

The nature of the MCP is even more controversial in two dimensions (2D) where different scenarios have been put forward; see, for example, Refs. 1,2,3,4,5,6,7,12,13,14,15,16. The existence of the Ising and the XY Kosterlitz-Thouless (KT) critical lines is well established. But, other features, like the existence of the spin-flop line, the nature of the MCP, and the MCP temperature, are still the object of debate. For example, the two different phase diagrams sketched in Fig. 4 have apparently been both supported by recent numerical Monte Carlo analyses: Ref. 15 claims that MC data are in agreement with the phase diagram 


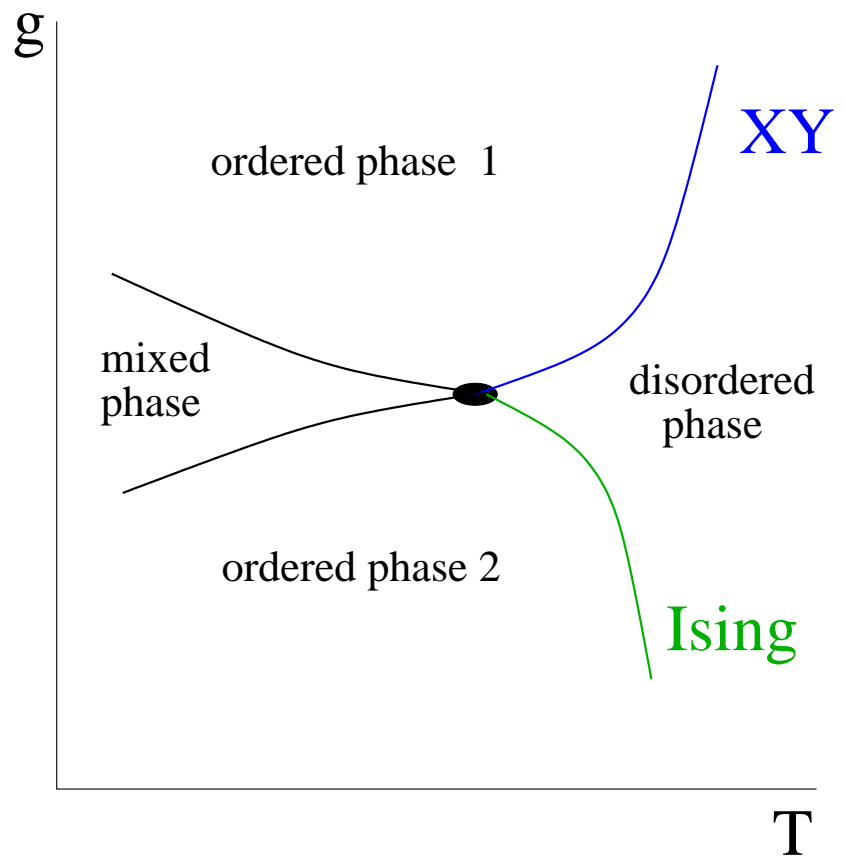

FIG. 2: Phase diagram with a tetracritical point where an Ising and an XY line meet. Here, $T$ is the temperature and $g$ a second relevant parameter.

reported on the left, while Ref. 16 supports that reported on the right. Similar contradictions appear in the analysis of the experimental data. ${ }^{1,2,3,4,5,6,7}$

In this paper we study the nature of the MCP in 2D within the field theoretical approach. Our main findings are the following:

(i) The MCP cannot be $\mathrm{O}(3)$ symmetric and cannot occur at $T=0$. Indeed, the magnetic field and the anisotropy give rise to an infinite number of relevant perturbations of the $\mathrm{O}(3)$ FP. Moreover, since at $T=0$ the order parameter is discontinuous as $H$ is varied, we expect a first-order spin-flop line. We thus predict a finite-temperature MCP and exclude a phase diagram such as the one shown in Fig. 4 on the right.

(ii) We study the limit of weak anisotropy. In this limit we determine how the critical temperature of the Ising and $\mathrm{KT}$ transition lines and of the MCP vary with $H$ and $A$.

Our results on the phase diagram of the classical XXZ model (1) are also relevant for the phase diagram of quantum spin- $S$ XXZ models ${ }^{17,18}$ and related systems, such as the hard-core boson Hubbard model. ${ }^{19,20}$

We finally mention that anisotropic antiferromagnets modelled by the XXZ model (1) on 


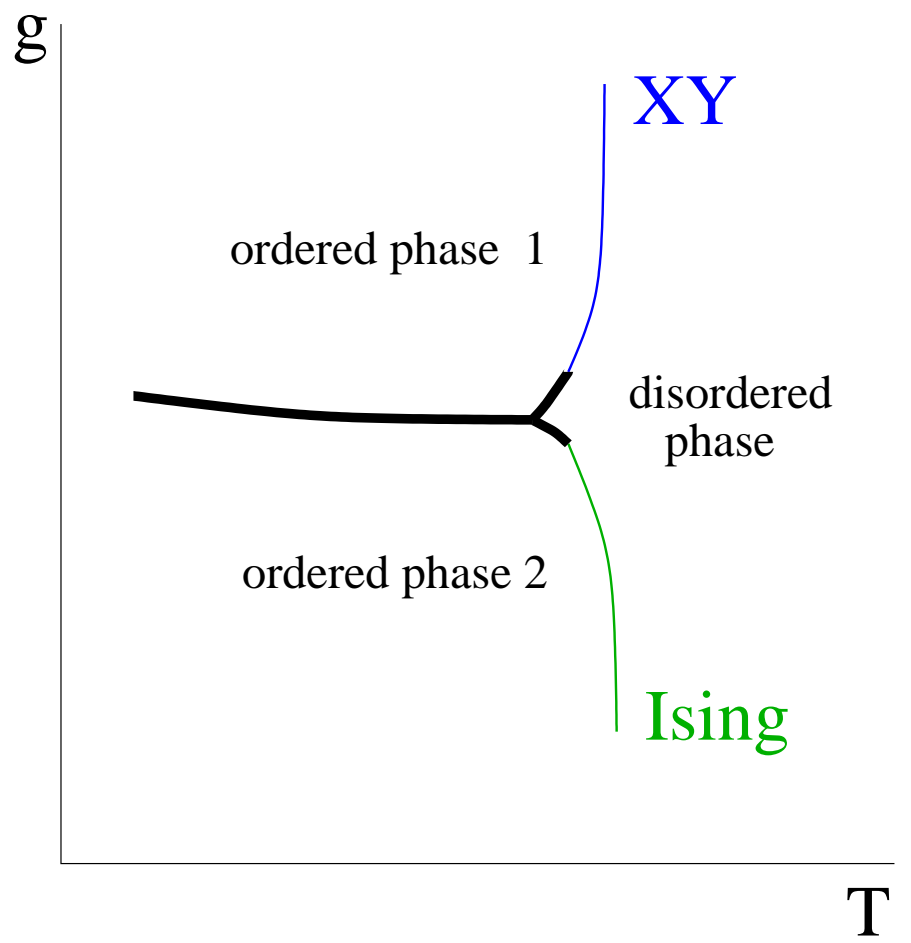

FIG. 3: Phase diagram with a bicritical point of first order. The thick black lines correspond to first-order transitions.

a triangular lattice or on a stacked triangular lattice (and, more generally, on lattices that are not bipartite) are expected to show a different multicritical behavior, essentially because of frustration. Ref. 21 reports a study of the possible phase diagrams in the mean-field approximation and a field-theory study of the 3D renormalization-group (RG) flow.

The paper is organized as follows. In Sec. II we summarize some general predictions for the isotropic model. In Sec. III we investigate the stability of the O(3) FP by using field theory and give predictions for the critical temperature as a function of $H$ and $A$ for small $A$ and $H$. Finally, in Sec. IV we discuss the implications of the field-theoretical results and discuss the possibile phase diagrams that are compatible with them.

\section{THE ISOTROPIC ANTIFERROMAGNET IN TWO DIMENSIONS}

For $H=A=0$, the critical behavior of model (1) is well known. Indeed, if we perform the change of variables

$$
\vec{\sigma}_{n}=(-1)^{[n]} \vec{S}_{n},
$$



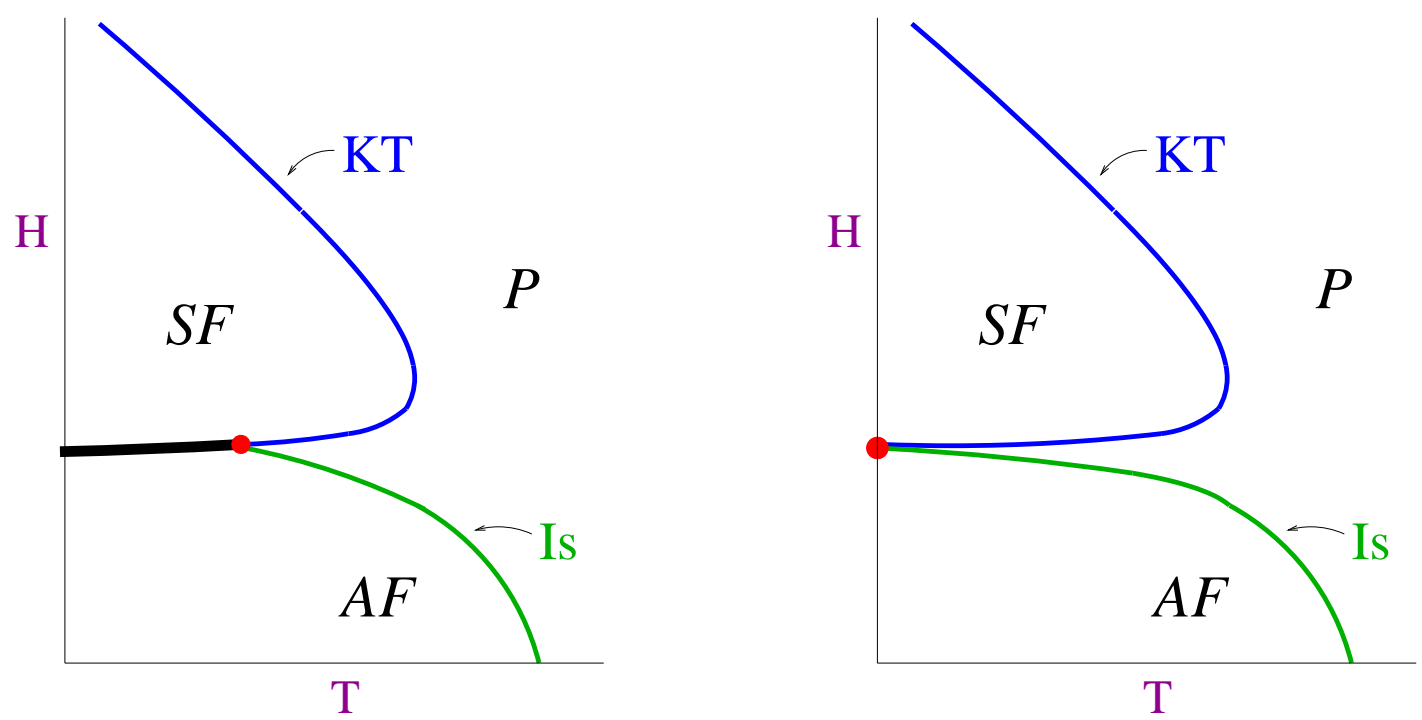

FIG. 4: Phase diagrams in the $T-H$ plane in the presence of easy-axis anisotropy $A>0$ in 2D. The left figure shows a first-order transition line separating the antiferromagnetic (AF) and spin-flop (SF) phases, with a bicritical point where the Ising (Is) and the XY Kosterlitz-Thouless (KT) critical lines meet. In the right figure the Ising and KT lines meet at a zero-temperature $\mathrm{O}(3)$-symmetric MCP.

where, in $2 \mathrm{D},[n] \equiv n_{x}+n_{y}\left[n=\left(n_{x}, n_{y}\right)\right]$, we obtain the ferromagnetic Heisenberg model for which several results are known. ${ }^{22}$ In particular, if we define the two-point function

$$
G(n)=\left\langle\vec{S}_{0} \cdot \vec{S}_{n}\right\rangle
$$

a universal critical behavior is observed for the staggered susceptibility

$$
\chi_{s}=\sum_{n}(-1)^{[n]} G(n)
$$

and for the staggered second-moment correlation length

$$
\xi_{s}^{2}=\frac{1}{4 \chi_{s}} \sum_{n}(-1)^{[n]}|n|^{2} G(n) .
$$

Under the mapping (4), $\chi_{s}$ and $\xi_{s}$ go over to the standard susceptibility and correlation length of the ferromagnetic model. In 2D, perturbation theory together with the RG allows one to derive the critical behavior of these two quantities for $T \rightarrow 0 .{ }^{23}$ Using the results of Refs. 24,25, we obtain

$$
\begin{aligned}
\chi_{s} & =C_{\chi} \xi_{s}^{2}\left(\frac{T}{2 \pi}\right)^{2}\left[1+0.18169 T+0.1334 T^{2}+0.1346 T^{3}+O\left(T^{4}\right)\right], \\
\xi_{s} & =C_{\xi} e^{2 \pi / T}\left(\frac{T}{2 \pi}\right)\left[1-0.0914 T-0.1969 T^{2}+O\left(T^{3}\right)\right] .
\end{aligned}
$$


The constants $C_{\xi}$ and $C_{\chi}$ cannot be determined in perturbation theory. Numerical values are reported in Ref. 26:

$$
C_{\chi}=93.25(3), \quad C_{\xi}=0.0124783(12)
$$

The asymptotic expansions (8) are quite accurate for $T \lesssim 0.3$, within a few percent at most. $^{27,28}$

\section{CRITICAL AND MULTICRITICAL BEHAVIOR FOR SMALL $A$ AND $H$}

\section{A. General results}

We consider the $\mathrm{O}(3)$ isotropic model at $H=0$ and add terms that break the $\mathrm{O}(3)$ symmetry down to $\mathbb{Z}_{2} \otimes \mathrm{O}(2)$ (for instance the magnetic field or the anisotropy). The corresponding general Hamiltonian is

$$
\mathcal{H}_{\text {gen }}=J \sum_{\langle n m\rangle} \vec{S}_{n} \cdot \vec{S}_{m}+\lambda Q(S)
$$

If $Q(S)$ is a relevant perturbation of the $\mathrm{O}(3) \mathrm{FP}$, the $\mathrm{O}(3)$ critical point is a MCP in the full theory. In $3 \mathrm{D}$ we can write the singular part of the free energy ${ }^{10}$ for $\lambda \rightarrow 0$ as

$$
\mathcal{F}_{\text {sing }} \sim u_{t}^{2-\alpha} B(X), \quad X=u_{\lambda} u_{t}^{-\phi},
$$

where $\alpha$ and $\phi$ are the $\mathrm{O}(3)$ specific-heat and crossover exponents, respectively, $B(X)$ is a universal scaling function, and $u_{t}$ and $u_{\lambda}$ are the scaling fields associated with the temperature and with $\lambda$. In general, we expect

$$
u_{t}=t+k \lambda
$$

where $k$ is a constant, $t \equiv T / T_{O(3)}-1$ is the reduced temperature, and $T_{O(3)}$ is the critical temperature of the isotropic model. No such mixing between $t$ and $\lambda$ occurs in $u_{\lambda}$, since $u_{\lambda}$ vanishes for $\lambda=0$. Hence, we can take $u_{\lambda}=\lambda$. The crossover exponent is related to the RG dimension $y_{\lambda}$ of the operator $Q$ that represents the perturbation of the MCP: $\phi \equiv y_{\lambda} \nu$. Suppose now that the system has a critical transition for $\lambda \neq 0$ at $T_{c}(\lambda)$. Since the singular part of the free energy close to a critical point behaves as $\left(T-T_{c}\right)^{2-\alpha}$, we must have $B\left(X_{c}\right)=0$, where $X_{c}$ is the value of $X$ obtained by setting $T=T_{c}(\lambda)$. This equation 
is solved by $X_{c}=X_{ \pm}$, where $X_{ \pm}$are two constants that depend on the sign of $\lambda$, such that $X_{+}>0, X_{-}<0$. Hence, we obtain

$$
\lambda\left[\frac{T_{c}(\lambda)}{T_{O(3)}}-1+k \lambda\right]^{-\phi}=X_{ \pm} .
$$

It follows

$$
\begin{array}{ll}
T_{c}(\lambda)=T_{O(3)}\left[1+\left(\lambda / X_{+}\right)^{1 / \phi}-k \lambda+\cdots\right], & \lambda>0, \\
T_{c}(\lambda)=T_{O(3)}\left[1+\left(\lambda / X_{-}\right)^{1 / \phi}-k \lambda+\cdots\right], & \lambda<0 .
\end{array}
$$

These expressions provide the $\lambda$ dependence of the critical temperature for $\lambda$ small. Note that, depending on the sign of $\lambda, T_{c}(\lambda)$ varies differently. The sign of $\lambda$ may also be relevant for the nature of the phase transition. Indeed, the low-temperature phase may be different depending on this sign: in this case one observes critical behaviors belonging to different universality classes for $\lambda>0$ and $\lambda<0$.

One can generalize these considerations to the case in which there are two relevant perturbations $Q_{1}$ and $Q_{2}$ with parameters $\lambda_{1}$ and $\lambda_{2}$. In this case we can write

$$
\mathcal{F}_{\text {sing }} \sim u_{t}^{2-\alpha} B\left(X_{1}, X_{2}\right),
$$

with

$$
\begin{aligned}
& X_{1} \equiv u_{1} u_{t}^{-\phi_{1}}, \quad X_{2} \equiv u_{2} u_{t}^{-\phi_{2}}, \\
& u_{1}=\lambda_{1}+c_{1} \lambda_{2}, \quad u_{2}=\lambda_{1}+c_{2} \lambda_{2}, \\
& u_{t}=t+k_{1} \lambda_{1}+k_{2} \lambda_{2},
\end{aligned}
$$

where $k_{1}, k_{2}, c_{1}$, and $c_{2}$ are constants. We have assumed here that there are two RG relevant operators that break the $\mathrm{O}(3)$ invariance, with $\mathrm{RG}$ dimensions $y_{1}>y_{2}>0$, as is the case for anisotropic systems in the presence of a magnetic field. The crossover exponents are $\phi_{1}=\nu y_{1}$ and $\phi_{2}=\nu y_{2}$. The scaling fields $u_{1}$ and $u_{2}$ are linear combinations (and, beyond linear order, generic functions) of $\lambda_{1}$ and $\lambda_{2}$, due to the fact that the lattice operators $Q_{1}$ and $Q_{2}$ generically couple both RG operators.

Let us now assume that, for $\lambda_{1}$ and $\lambda_{2}$ small, the model shows generically two types of phase transitions belonging to different universality classes and that, for specific values of the ratio $\lambda_{1} / \lambda_{2}$, multicritical transitions occur. As before, we wish to derive the dependence 
of the critical temperature as a function of $\lambda_{1}$ and $\lambda_{2}$ for $\lambda_{1}, \lambda_{2} \rightarrow 0$. Since

$$
X_{2}=u_{2}\left|u_{1}\right|^{-\phi_{2} / \phi_{1}}\left|X_{1}\right|^{\phi_{2} / \phi_{1}}
$$

we can rewrite Eq. (15) as

$$
\mathcal{F}_{\text {sing }} \sim u_{t}^{2-\alpha} \mathcal{B}_{ \pm}\left(X_{1}, u_{2}\left|u_{1}\right|^{-\phi_{2} / \phi_{1}}\right)
$$

where we have introduced two different functions depending on the sign of $u_{1}$. If $u_{1}>0$ the relevant function is $\mathcal{B}_{+}(x, y)$, if $u_{1}<0$ one should consider $\mathcal{B}_{-}(x, y)$. At the critical point we must have

$$
\mathcal{B}_{ \pm}\left(X_{1 c}, u_{2}\left|u_{1}\right|^{-\phi_{2} / \phi_{1}}\right)=0
$$

which implies

$$
X_{1 c}=F_{ \pm}\left(u_{2}\left|u_{1}\right|^{-\phi_{2} / \phi_{1}}\right),
$$

with $F_{+}(x)>0$ and $F_{-}(x)<0$. We obtain finally

$$
\frac{T_{c}}{T_{O(3)}}-1 \approx\left[\frac{u_{1}}{F_{ \pm}\left(u_{2}\left|u_{1}\right|^{-\phi_{2} / \phi_{1}}\right)}\right]^{1 / \phi_{1}}-k_{1} \lambda_{1}-k_{2} \lambda_{2} .
$$

Let us now discuss some limiting cases. First, assume that $u_{2}\left|u_{1}\right|^{-\phi_{2} / \phi_{1}} \ll 1$. This implies that $X_{2}$ is small compared to $X_{1}$, so that we can neglect $X_{2}$. We are back to the case considered before and thus we can use Eq. (14). Depending on the sign of $u_{1}$ we have:

1) If $u_{1}>0$, the phase transition is located at

$$
\frac{T_{c}}{T_{O(3)}}-1 \approx\left(\frac{u_{1}}{X_{+}}\right)^{1 / \phi_{1}}-k_{1} \lambda_{1}-k_{2} \lambda_{2}
$$

where $X_{+}>0$.

2) If $u_{1}<0$, there is a phase transition located at

$$
\frac{T_{c}}{T_{O(3)}}-1 \approx\left(\frac{u_{1}}{X_{-}}\right)^{1 / \phi_{1}}-k_{1} \lambda_{1}-k_{2} \lambda_{2}
$$

where $X_{-}<0$.

Comparing with Eq. (21) we obtain $F_{ \pm}(0)=X_{ \pm}$.

The second interesting limiting case corresponds to $u_{1} \rightarrow 0$. In this case $X_{1}$ can be neglected and we need to consider only $X_{2}$. Therefore, depending on the sign of $u_{2}$, we have a MCP located at

$$
\frac{T_{c}}{T_{O(3)}}-1 \approx\left(\frac{u_{2}}{X_{\mathrm{mc}, \pm}}\right)^{1 / \phi_{2}}-k_{1} \lambda_{1}-k_{2} \lambda_{2}
$$


where $X_{\mathrm{mc},+}>0$ and $X_{\mathrm{mc},+}<0$. Consistency with Eq. (21) requires

$$
\begin{array}{ll}
F_{ \pm}(x) \approx \pm\left(x / X_{\mathrm{mc},+}\right)^{-\phi_{1} / \phi_{2}} & \text { for } x \rightarrow+\infty \\
F_{ \pm}(x) \approx \pm\left(x / X_{\mathrm{mc},-}\right)^{-\phi_{1} / \phi_{2}} & \text { for } x \rightarrow-\infty .
\end{array}
$$

One may devise simple interpolations that are exact for $x \rightarrow 0$ and $x \rightarrow \infty$. If, for instance, $u_{1}>0$ and $u_{2}>0$, we may consider the approximate expression

$$
\frac{T_{c}}{T_{O(3)}}-1 \approx\left(\frac{u_{1}+b u_{2}^{\phi_{1} / \phi_{2}}}{X_{+}}\right)^{1 / \phi_{1}}-k_{1} \lambda_{1}-k_{2} \lambda_{2}, \quad b \equiv X_{+} X_{\mathrm{mc},+}^{-\phi_{1} / \phi_{2}}
$$

The functions $F_{ \pm}(x)$ are crossover functions that interpolate between the two regimes in which only one of the relevant operators is present. As far as the nature of the transition, the relevant quantity is the sign of $u_{1}$. In general, we expect that the transition belongs to different universality classes depending on the sign of $u_{1}$. For $u_{1}=0$ the leading relevant operator decouples and thus we obtain a MCP whose nature may depend on the sign of $u_{2}$.

The previous results apply to the 3D model but cannot be used directly in $2 \mathrm{D}$, since in this case $T_{O(3)}=0$ and $\nu$ is not defined $\left(\xi_{s} \sim e^{2 \pi / T}\right.$ for $\left.\lambda=0\right)$. To investigate the $2 \mathrm{D}$ case, let us consider again Hamiltonian (10), let us assume that $Q(S)$ renormalizes multiplicatively under RG transformations and that its RG dimension is 2 with logarithmic corrections (as we shall see, this is the case of interest). The perturbative analysis of the scaling behavior of the free energy is analogous to that presented in Ref. 29. The correct scaling variable is

$$
Y=u_{\lambda}\left(\frac{T}{2 \pi}\right)^{p} e^{4 \pi / T} \sim u_{\lambda}\left(\frac{T}{2 \pi}\right)^{p-2} \xi_{s}^{2}
$$

where we used Eq. (8), and $p$ is a power that can be computed by using the one-loop expression of the anomalous dimension of $Q$. Then, the singular part of the free energy is given by

$$
\mathcal{F}_{\text {sing }} \approx \xi_{s}^{-2} B(Y)
$$

The critical line is again characterized by $Y=Y_{ \pm}$, i.e. by

$$
\lambda\left(\frac{T_{c}}{2 \pi}\right)^{p} e^{4 \pi / T_{c}}=Y_{ \pm}
$$

with $Y_{+}>0$ and $Y_{-}<0$. Solving this equation for $T_{c}$, we obtain

$$
T_{c}=\frac{4 \pi}{\ln \left(Y_{ \pm} / \lambda\right)}\left[1-p \frac{\ln \frac{1}{2} \ln \left(Y_{ \pm} / \lambda\right)}{\ln \left(Y_{ \pm} / \lambda\right)}+\cdots\right]
$$


The discussion is analogous in the case there are two relevant perturbations. We define two scaling variables $Y_{1}$ and $Y_{2}$ as

$$
Y_{i}=u_{i}\left(\frac{T}{2 \pi}\right)^{p_{i}} e^{4 \pi / T}, \quad i=1,2,
$$

and write the free energy as

$$
\mathcal{F}_{\text {sing }}=\xi_{s}^{-2} B\left(Y_{1}, Y_{2}\right)
$$

If $p_{1}<p_{2}$, the critical behavior depends on the sign of $u_{1}$ : For $u_{1}>0$ and $u_{1}<0$ one

obtains different critical behaviors. In the limit in which $Y_{2}$ is small and can be neglected, we can write

$$
T_{c}=\frac{4 \pi}{\ln \left(Y_{ \pm} / u_{1}\right)}\left[1-p_{1} \frac{\ln \frac{1}{2} \ln \left(Y_{ \pm} / u_{1}\right)}{\ln \left(Y_{ \pm} / u_{1}\right)}\right],
$$

where $Y_{ \pm}$are two constants such that $Y_{+}>0$ and $Y_{-}<0$. As observed in the 3D case, Eq. (33) [it is the analogue of Eqs. (22) and (23)] holds only if $Y_{2}$ can be neglected. Since

$$
Y_{2}=\frac{u_{2}}{u_{1}} Y_{1}\left(\frac{T}{2 \pi}\right)^{p_{2}-p_{1}} \approx \frac{u_{2}}{u_{1}} Y_{1}\left(\frac{1}{2} \ln \left(Y_{1} / u_{1}\right)\right)^{p_{1}-p_{2}}
$$

Eq. (33) holds only in the parameter region in which

$$
u_{2} \ll u_{1}\left(\frac{1}{2} \ln \left(Y_{ \pm} / u_{1}\right)\right)^{p_{2}-p_{1}}
$$

i.e. far from the MCP at $u_{1}=0$. For $\left|u_{1}\right| \rightarrow 0$ this region shrinks to zero and thus a correct formula requires the full crossover function.

For $u_{1}=0$, we have a MCP at

$$
T_{c}=\frac{4 \pi}{\ln \left(Y_{\mathrm{mc}, \pm} / u_{2}\right)}\left[1-p_{2} \frac{\ln \frac{1}{2} \ln \left(Y_{\mathrm{mc}, \pm} / u_{2}\right)}{\ln \left(Y_{\mathrm{mc}, \pm} / u_{2}\right)}\right],
$$

depending on the sign of $u_{2}$.

\section{B. Effective Hamiltonians}

Let us now apply the results of the previous section to the XXZ model. For this purpose we consider the ferromagnetic Hamiltonian corresponding to (1) under the mapping (4),

$$
\mathcal{H}_{f}=-\sum_{\langle n m\rangle} \vec{\sigma}_{n} \cdot \vec{\sigma}_{m}-A \sum_{\langle n m\rangle} \sigma_{n, z} \sigma_{m, z}+H \sum_{n}(-1)^{[n]} \sigma_{n, z}
$$


where we have set $J=1$ for convenience. We argue that Hamiltonian (37) is equivalent to the Hamiltonian

$$
\mathcal{H}^{*}=-\sum_{\langle n m\rangle} \vec{\sigma}_{n} \cdot \vec{\sigma}_{m}+\sum_{l \geq 1} \alpha_{l}(T, h, a) \mathcal{O}_{2 l}(\sigma),
$$

where $\mathcal{O}_{2 l}(\sigma)$ are the zero-momentum dimension-zero spin-2l perturbations of the $\mathrm{O}(3) \mathrm{FP}$, $h=H / T, a=A / T$ (note that the partition function depends on $h$ and $a$ ). The operators $\mathcal{O}_{2 l}(\sigma)$ can be constructed starting from the symmetric traceless operators of degree $2 l$, see, e.g., Ref. 30. Explicitly, for $l=1,2$ we have

$$
\begin{aligned}
& \mathcal{O}_{2}(\sigma)=\sum_{n}\left(\sigma_{n, z}^{2}-1 / 3\right), \\
& \mathcal{O}_{4}(\sigma)=\sum_{n}\left(\sigma_{n, z}^{4}-\frac{6}{7} \sigma_{n, z}^{2}+\frac{3}{35}\right) .
\end{aligned}
$$

The functions $\alpha_{l}(T, h, a)$ are smooth and vanish for $h \rightarrow 0$ and $a \rightarrow 0$. It should be stressed that the two Hamiltonians are equivalent only for the computation of the leading critical behavior of long-distance quantities.

The equivalence of $\mathcal{H}_{f}$ and $\mathcal{H}^{*}$ for what concerns the critical behavior can be justified on the basis of the results of Ref. 10 obtained in the usual LGW approach. In the absence of anisotropy and magnetic field, i.e. for $A=H=0$, model (1) is $\mathrm{O}(3)$ invariant and thus its critical behavior is described by the usual LGW Hamiltonian

$$
\mathcal{H}_{\Phi^{4}}=\int d^{d} x\left[\frac{1}{2} \sum_{\mu}\left(\partial_{\mu} \Phi\right)^{2}+\frac{r}{2} \Phi^{2}+\frac{u}{4 !} \Phi^{4}\right]
$$

where $\vec{\Phi}$ is a three-component vector. The magnetic field and the anisotropy break the $\mathrm{O}(3)$ invariance. If we define $\vec{\Phi}=(\vec{\phi}, \varphi)$, where $\vec{\phi}$ is a two-component vector and $\varphi$ a scalar, the LGW Hamiltonian (3) corresponding to (1) can be written as ${ }^{10}$

$$
\left.\mathcal{H}=H_{\Phi^{4}}+\int d^{d} x\left[f_{22} P_{22}+f_{42} P_{42}+f_{44} P_{44}\right)\right]
$$

where

$$
\begin{aligned}
& P_{22}=\varphi^{2}-\frac{1}{3} \Phi^{2} \\
& P_{42}=\Phi^{2} P_{22} \\
& P_{44}=\varphi^{4}-\frac{6}{7} \Phi^{2} \varphi^{2}+\frac{3}{35}\left(\Phi^{2}\right)^{2},
\end{aligned}
$$


and $f_{i j}$ are coupling constants depending on $a, h^{2}$, and $T$. We have introduced here the homogeneous polynomial $P_{m l}$. The polynomial $P_{l l}$ has degree $l$ and transforms irreducibly as a spin- $l$ representation of the $\mathrm{O}(3)$ group. ${ }^{31}$ Polynomials $P_{m l}, m>l$, are defined as $P_{m l} \equiv\left(\Phi^{2}\right)^{(m-l) / 2} P_{l l}$. The classification of the zero-momentum perturbations in terms of spin values is particularly convenient, since polynomials with different spin do not mix under RG transformations and the RG dimensions $y_{m l}$ do not depend on the particular component

of the spin-l representation. We refer to Ref. 8 for details. Thus, in the LGW approach the $\mathrm{O}(3)$ Hamiltonian is perturbed by spin-2 and spin-4 perturbations. If one considers higher power of the fields, also spin-6, spin-8, ... perturbations appear. They are irrelevant in $3 \mathrm{D}$, but must be considered in $2 \mathrm{D}$, since in this case the field $\Phi$ is dimensionless and any polynomial of the fields is relevant.

\section{Three-dimensional behavior}

In $3 \mathrm{D}$ the critical behavior is well known:

(i) In the absence of anisotropy, i.e. for $A=0$, there is an XY critical line $T_{X Y}(H)$ ending at the $\mathrm{O}(3)$ critical point for $H=0$. The phase diagram is symmetric under $H \rightarrow-H$.

(ii) In the absence of magnetic field, i.e. for $H=0$, there are two critical lines: an XY critical line for $A<0$ and an Ising critical line for $A>0$, which meet at the $\mathrm{O}(3)$ critical point as $A \rightarrow 0$.

(iii) If $A$ and $H$ are both present, one observes two different phase diagrams depending on the sign of $A$. If $A$ is negative, there is an XY transition. If $A$ is positive, there is an Ising critical line for $H$ small and an XY critical line for $H$ large; the two lines meet at a MCP corresponding to a nonvanishing value of $H$.

We wish now to determine the dependence of the critical temperatures on $A$ and $H$ close to the $\mathrm{O}(3)$ point. For this purpose we investigate the relevance of the perturbations. In $3 \mathrm{D}$ we expect that only polynomials of degree two or four are relevant. Thus, $P_{22}, P_{42}$ and $P_{44}$ are the only quantities that should be considered. The RG analysis is reported in Ref. 8 and indicates that $P_{42}$ is irrelevant, while $P_{22}$ and $P_{44}$ are relevant. Perturbative fieldtheory calculations provide estimates of the corresponding RG dimensions: ${ }^{8,32} y_{22} \approx 1.79$, 


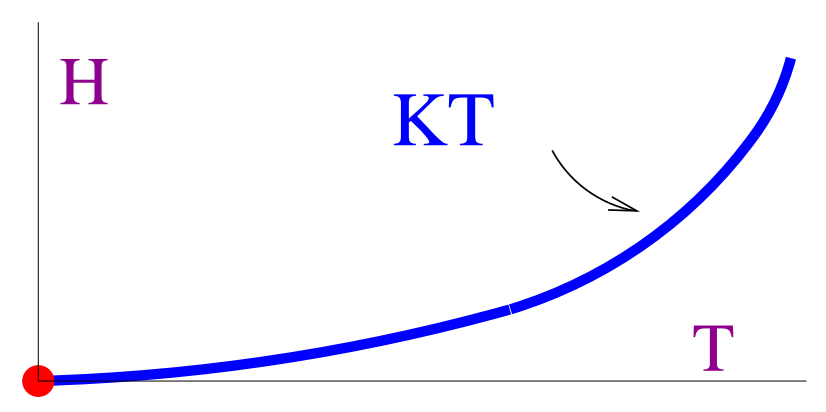

FIG. 5: Phase diagram of isotropic antiferromagnets in the $T$ - $H$ plane.

$y_{44} \approx 0.01$, and $y_{42} \approx-0.55$. The relevant scaling fields are $u_{1}=a+c_{1} h^{2}$ associated with $P_{22}$ and $u_{2}=a+c_{2} h^{2}$ associated with $P_{44}$. The scaling fields depend on $h^{2}$, because of the symmetry under $H \rightarrow-H$ transformations. Since $P_{22}$ is the most relevant operator, the critical behavior depends on the sign of $u_{1}$. We obtain XY behavior for $a+c_{1} h^{2}<0$ and Ising behavior for $a+c_{1} h^{2}>0$. Since for $A=0$ only the XY transition is observed, the constant $c_{1}$ must be negative. Hence, Ising behavior can only be observed for $a$ positive and $h^{2}$ not too large, in agreement with experiments. The critical temperatures behave as

$$
\begin{gathered}
T_{c, \mathrm{XY}}-T_{O(3)}=b_{\mathrm{XY}}\left(-a-c_{1} h^{2}\right)^{1 / \phi_{22}}-k_{1} a-k_{2} h^{2} \\
T_{c, \mathrm{Is}}-T_{O(3)}=b_{\mathrm{Is}}\left(a+c_{1} h^{2}\right)^{1 / \phi_{22}}-k_{1} a-k_{2} h^{2}
\end{gathered}
$$

where $b_{\mathrm{XY}}, b_{\mathrm{Is}}, k_{1}$ and $k_{2}$ are constants, with $b_{\mathrm{XY}}>0, b_{\mathrm{Is}}>0 . \operatorname{Here}^{8} \phi_{22}=1.260(11)$, $1 / \phi_{22}=0.794(7)$.

Multicritical behavior is observed for $a=-c_{1} h^{2}$ (since $c_{1}$ is negative this equality can only occur for $a>0)$. Since $1 / \phi_{44} \gtrsim 100$, the leading nonanalytic term with exponent $1 / \phi_{44}$ cannot be observed in practice, and therefore

$$
T_{c, \mathrm{mc}}-T_{O(3)}=-k_{1} a-k_{2} h^{2}
$$

\section{Two-dimensional behavior}

The critical behavior in 2D is analogous to that observed in 3D. For $A<0$ there is an $\mathrm{XY}$ Kosterlitz-Thouless (KT) transition for any $H$, while for $A>0$ there is an Ising transition for small $|H|$, an XY transition for large $|H|$, and a MCP in between. The phase diagram in the two limiting cases $A=0$ and $H=0$ is reported in Figs. 5 and 6 . 


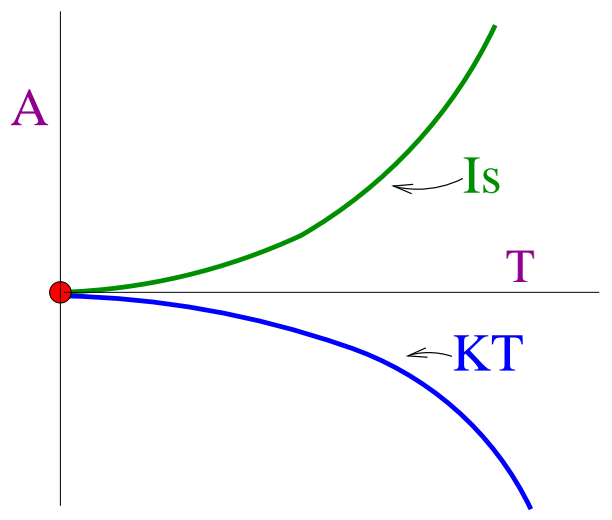

FIG. 6: Phase diagram of anisotropic antiferromagnets in the $T-A$ plane for $H=0$.

To compute the position of the critical point, we should investigate the relevance of the perturbations $P_{m l}$. As shown in Ref. 33, in 2D any spin- $l$ perturbation $P_{l l}$ is relevant at the $\mathrm{O}(3) \mathrm{FP}$, since the corresponding $\mathrm{RG}$ dimension $y_{l l}$ is positive, indeed $y_{l l}=2$ apart from logarithms which can be computed by using perturbation theory. One can also argue that perturbations $P_{m l}$ with $m>l$ can be neglected. Indeed, spin waves, that are rotations of the spins, are the critical modes of the ferromagnetic $\mathrm{O}(3)$ model. Changes in the size of the field $\Phi$ should not be critical. Therefore, $P_{m l} \equiv\left(\Phi^{2}\right)^{(m-l) / 2} P_{l l}$ should be equivalent to $\left(\Phi_{0}^{2}\right)^{(m-l) / 2} P_{l l} \sim P_{l l}$, where $\Phi_{0}^{2}$ is the average of $\Phi^{2}$. Thus, one should only consider the operators $P_{l l}$. Equation (38) then follows immediately. The equivalence of model (1) with Hamiltonian (38) was already conjectured in Ref. 34, even though there only the leading spin-2 term was explicitly considered.

The relevant scaling variables are

$$
Y_{2 l}=u_{l}\left(\frac{T}{2 \pi}\right)^{l(2 l+1)+2} e^{4 \pi / T},
$$

which are associated with the spin- $2 l$ perturbation. The power of $T$, which is universal, has been determined by using the perturbative results of Ref. 33, which provide the anomalous dimension of any zero-dimension spin- $l$ perturbation of the $2 \mathrm{D} N$-vector model. The scaling fields $u_{l}$ are linear combinations of the parameters that break the $\mathrm{O}(3)$ invariance. As before, we write them as $u_{l}=a+c_{l} h^{2}$. As a consequence, the free energy can be written as

$$
\mathcal{F}_{\text {sing }} \approx \xi^{-2} \hat{F}\left(Y_{2}, Y_{4}, \ldots\right) .
$$

Note that different powers of $T$ appear in the definition (45). The most relevant term for $T \rightarrow 0$ corresponds to the spin- 2 operator, since $Y_{2 l} \sim Y_{2} T^{l(2 l+1)-3}$ and $l(2 l+1)-3$ is 
positive for $l \geq 2$. Next one should consider the spin-4 scaling variable $Y_{4}$. If one considers the scaling limit at fixed $Y_{2} \neq 0$ or $Y_{4} \neq 0$, the higher-order spin variables go to zero as $T \rightarrow 0$ and thus represent corrections to scaling proportional to powers of $T$. Thus, in the scaling limit one can neglect $Y_{6}, Y_{8}, \ldots$, and write

$$
\mathcal{F}_{\text {sing }} \approx \xi^{-2} F_{2}\left(Y_{2}, Y_{4}\right)
$$

which coincides with Eq. (32). We can thus use the above-obtained results.

(i) For $u_{1}<0$, i.e. $a+c_{1} h^{2}<0$, there is a KT transition at

$$
T_{c, \mathrm{XY}}=\frac{4 \pi}{\ln \left(Y_{\mathrm{XY}} / u_{1}\right)}\left[1-5 \frac{\ln \frac{1}{2} \ln \left(Y_{\mathrm{XY}} / u_{1}\right)}{\ln \left(Y_{\mathrm{XY}} / u_{1}\right)}\right],
$$

where $Y_{\mathrm{XY}}<0$. Note that the occurence of an XY transition for $a=0$ implies $c_{1}<0$ as in the $3 \mathrm{D}$ case.

(ii) For $u_{1}>0$, i.e. $a+c_{1} h^{2}>0$, there is an Ising transition at

$$
T_{c, \text { Is }}=\frac{4 \pi}{\ln \left(Y_{\text {Is }} / u_{1}\right)}\left[1-5 \frac{\ln \frac{1}{2} \ln \left(Y_{\text {Is }} / u_{1}\right)}{\ln \left(Y_{\text {Is }} / u_{1}\right)}\right],
$$

where $Y_{\text {Is }}>0$. Since $c_{1}<0$ this transition can only occur for $a>0$ and $h^{2}$ small.

(iii) For $u_{1}=0$, i.e. $a+c_{1} h^{2}=0$, there is a MCP at

$$
T_{c, \mathrm{mc}}=\frac{4 \pi}{\ln \left(Y_{\mathrm{mc}} / h^{2}\right)}\left[1-12 \frac{\ln \frac{1}{2} \ln \left(Y_{\mathrm{mc}} / h^{2}\right)}{\ln \left(Y_{\mathrm{mc}} / h^{2}\right)}\right],
$$

where $Y_{\mathrm{mc}}>0$. Note that since the MCP occurs for $a=-c_{1} h^{2}$, we can replace $u_{2}=a+c_{2} h^{2}$ simply with $h^{2}$.

Note that, as discussed before, Eqs. (48) and (49) are valid only as long as condition (35) is satisfied, i.e. far from the MCP $u_{1}=0$.

\section{DISCUSSION AND CONCLUSIONS}

In Sec. IIID we have discussed the behavior of the classical 2D XXZ model (1) on a square lattice in the presence of a magnetic field along the easy axis, for $A$ and $H$ small, focusing on the dependence of the critical temperature on $A$ and $H$. In this section we wish to discuss the possible scenarios for the nature of the MCP. 


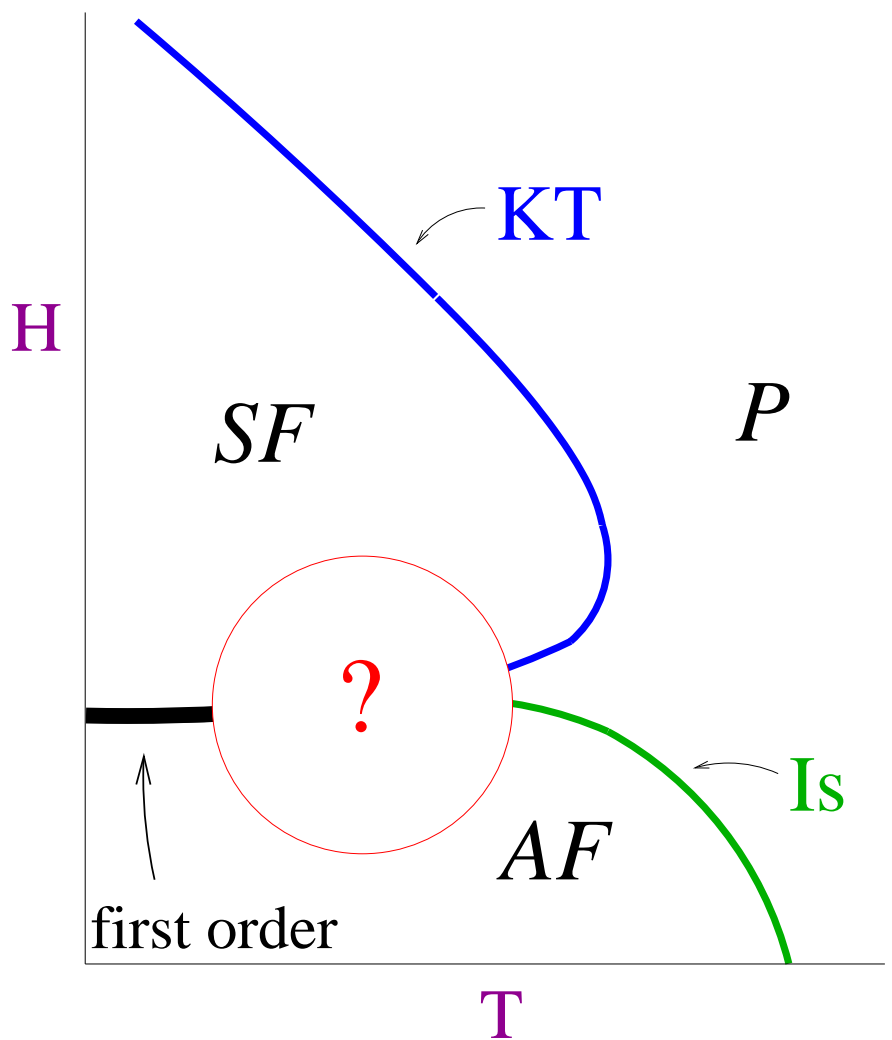

FIG. 7: Phase diagram in the $T-H$ plane in the presence of uniaxial anisotropy $A>0$.

First, we exclude the possibility that the MCP is $\mathrm{O}(3)$ symmetric and located at $T=0$, and thus we exclude a phase diagram such as the one shown on the right in Fig. 4 . This is implicit in the results of Sec. IIID, but we wish to present here an extended discussion. There are essentially two observations that exclude an $\mathrm{O}(3) \mathrm{MCP}$.

- For $T=0$ the XXZ model (1) on a square lattice can be easily solved. ${ }^{12,35}$ At fixed $A>0$, one finds two critical values of the magnetic field:

$$
\begin{aligned}
& H_{c 1}=4 J \sqrt{2 A+A^{2}}, \\
& H_{c 2}=4 J(2+A) .
\end{aligned}
$$

For $|H|<H_{c 1}$ the system is in a fully aligned antiferromagnetic configuration, for $H_{c 1}<|H|<H_{c 2}$ the system is in a spin-flop configuration, while for $|H|>H_{c 2}$ all spins are aligned with the magnetic field. The spin-flop transition at $|H|=H_{c 1}$ is of first order, since the order parameter, the staggered magnetization, has a discontinuity. If the transition were at $T=0$, the MCP should coincide with the spin-flop point $|H|=H_{c 1}$. Thus, the magnetization, the susceptibility and all critical quantities 


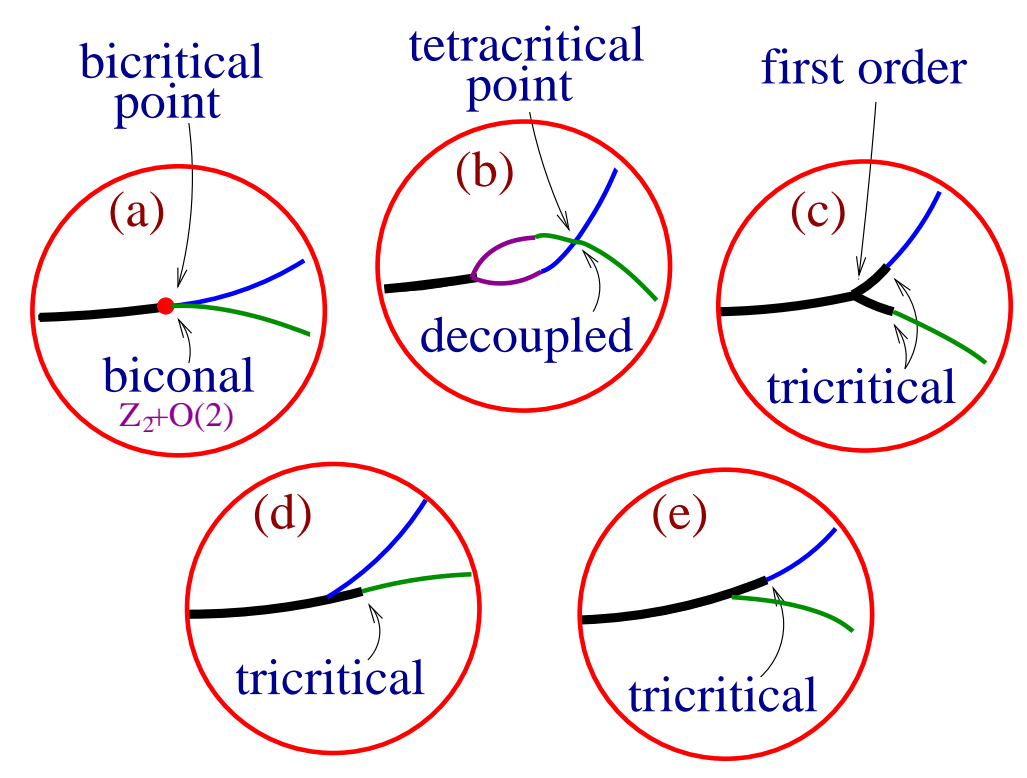

FIG. 8: Some possible phase diagrams inside the blob appearing in Fig. 7.

would have a discontinuity at $T=0$ when varying $H$. It is unclear how an $\mathrm{O}(3)$ critical behavior might be consistent with this discontinuity. On the other hand, the first-order behavior at $T=0$ is consistent with the existence of a first-order spin-flop line.

- The second objection is based on the LGW analysis presented in Sec. III B. The O(3) FP is unstable under an infinite number of perturbations. Thus, an infinite number of tunings is needed to recover the $\mathrm{O}(3)$ symmetry.

On the basis of these remarks, in 2D anisotropic antiferromagnets in a magnetic field along the easy axis, a scenario based on a $T=0 \mathrm{O}(3)$-symmetric MCP appears untenable. These conclusions are analogous to those that hold in $3 \mathrm{D} .^{8,9}$ It should be noted that in $2 \mathrm{D}$ the argument is much stronger. While in 3D these conclusions are based on the numerical determination of the RG dimension of the spin- 4 perturbation, in 2D they follow from the relevance of the spin- $l$ perturbations, which is an exact result. Moreover, while in 3D the $\mathrm{O}(3)$ behavior can be obtained by tuning a single additional parameter in such a way to decouple both the spin- 2 and the spin- 4 operator, in $2 \mathrm{D}$ the $\mathrm{O}(3)$ behavior can never be obtained at finite $A$, since an infinite number of tunings is needed.

Since we have proved that in 2D the MCP cannot have $\mathrm{O}(3)$ symmetry, an open question concerns the nature of the MCP. We shall now show that the decoupled FP, corresponding 
to a multicritical behavior in which the two order parameters are effectively uncoupled, is stable. The stability of the decoupled FP can be proved by nonperturbative arguments. ${ }^{36}$ Indeed, the RG dimension $y_{w}$ of the operator $\Phi_{1}^{2} \Phi_{2}^{2}$ that couples the two order parameters in the LGW theory (3) is given by

$$
y_{w}=\frac{1}{\nu_{\mathrm{KT}}}+\frac{1}{\nu_{\mathrm{Is}}}-2=-1<0 .
$$

since $\nu_{\mathrm{KT}}=\infty$ and $\nu_{\mathrm{Is}}=1$. Therefore, the perturbation is irrelevant and the decoupled FP is stable. Note that a decoupled MCP is always tetracritical, as in Fig. 2.

The stability of the decoupled FP and the instability of the $\mathrm{O}(3) \mathrm{FP}$ is also consistent with some general arguments. ${ }^{37}$ At a MCP the exponent $\eta$ describing the critical behavior of the correlation function of the order parameter is replaced by a matrix $\eta_{i j}$, see, e.g., Ref. 10 . The conjecture of Ref. 37 states that Tr $\eta$ should have a maximum at the stable FP. This indeed occurs in the present case: at the decoupled FP we have $\operatorname{Tr} \eta=3 / 4$ while at the $\mathrm{O}(3) \mathrm{FP} \operatorname{Tr} \eta=0$.

In Fig. 7 we show a plausible phase diagram, with three transition lines: a spin-flop firstorder transition line, an Ising and a KT critical line. The phase diagram inside the blob is an open issue. Some possibilities are shown in Fig. 8:

- Fig. 8 (a) presents a bicritical point, which may be associated with a stable biconal FP, whose attraction domain is in the bicritical region of the bare parameters of the LGW $\Phi^{4}$ theory. We should say that we do not have any evidence for the existence of such a FP.

- In Fig. 8 (b) we show a tetracritical point, where the Ising and KT lines intersect each other. In this case the MCP may be controlled by the stable decoupled FP discussed above.

- In Fig. 8 (c) the transition at the MCP is of first order. Starting from the MCP, the first-order transitions extend up to tricritical points, where the Ising and the KT critical lines start. This occurs if the system is outside the attraction domain of the stable FP of the RG flow. This scenario resembles the one predicted in 3D, see Fig. 3.

- Finally, we cannot exclude phase diagrams like those shown in Fig. 8 (d) and Fig. 8 (e). Case (d) is apparently observed in antiferromagnets with single-ion anisotropy 
and more than nearest-neighbor interactions, ${ }^{38}$ and also in hard-core boson systems, ${ }^{20}$ which are equivalent to anisotropic spin-1/2 XXZ systems in a magnetic field.

Of course, further experimental and theoretical investigations are called for to conclusively settle this issue.

\section{Acknowledgments}

We thank Pasquale Calabrese for useful discussions.

1 L.J. de Jongh, L.P. Regnault, J. Rossat Mignod, and J.Y. Henry, J. Appl. Phys. 53, 7963 (1982).

2 L.J. de Jongh and H.J.M. de Groot, Solid State Commun. 53, 737 (1985).

3 H.J.M. de Groot and L.J. de Jongh, Physica B \& C 141, 1 (1986).

4 H. Rauh, W.A.C. Erkelens, L.P. Regnault, J. Rossad-Mignod, W. Kullmann, and R. Geick, J. Phys. C 19, 4503 (1986).

5 R.A. Cowley, A. Aharony, R.J. Birgeneau, R.A. Pelcovits, G. Shirane, and T.R. Thurston, Z. Phys. B: Condens. Matter 93, 5 (1993).

6 R. van de Kamp, M. Steiner, and H. Tietze-Jaensch, Physica B 241, 570 (1998).

7 R.J. Christianson, R.L. Leheny, R.J. Birgeneau, and R.W. Erwin, Phys. Rev. B 63, 140401(R) (2001).

8 P. Calabrese, A. Pelissetto, and E. Vicari, Phys. Rev. B 67, 054505 (2003).

9 M. Hasenbusch, A. Pelissetto, and E. Vicari, Phys. Rev. B 72, 014532 (2005).

10 M.E. Fisher and D.R. Nelson, Phys. Rev. Lett. 32, 1350 (1974); D.R. Nelson, J.M. Kosterlitz, and M.E. Fisher, Phys. Rev. Lett. 33, 813 (1974); J.M. Kosterlitz, D.R. Nelson, and M.E. Fisher, Phys. Rev. B 13, 412 (1976).

11 K.-S. Liu and M. E. Fisher, J. Low Temp. Phys. 10, 655 (1972).

12 D.P. Landau and K. Binder, Phys. Rev. B 17, 2328 (1978).

13 D.P. Landau and K. Binder, Phys. Rev. B 24, 1391 (1981).

14 B.V. Costa and A.S.T. Pires, J. Magn. Magn. Mater. 262, 316 (2003).

15 M. Holtschneider, W. Selke, and R. Leidl, Phys. Rev. B 72, 064443 (2005).

16 C. Zhou, D.P. Landau, and T.C. Schulthess, Phys. Rev. B 74, 064407 (2006). 
17 A. Cuccoli, T. Roscilde, V. Tognetti, R. Vaia, and P. Verrucchi, Eur. Phys. J B 20, 55 (2001).

18 A. Cuccoli, T. Roscilde, V. Tognetti, R. Vaia, and P. Verrucchi, Phys. Rev. B 67, 104414 (2003).

19 F. Hébert, G.G. Batrouni, R.T. Scalettar, G. Schmid, M. Troyer, and A. Dorneich, Phys. Rev. B 65, 014513 (2001).

20 G. Schmid, S. Todo, M. Troyer, and A. Dorneich, Phys. Rev. Lett. 88, 167208 (2002).

21 P. Calabrese, A. Pelissetto, and E. Vicari, Nucl. Phys. B 709, 550 (2005).

22 A. Pelissetto and E. Vicari, Phys. Rep. 368, 549 (2002).

23 J. Zinn-Justin, Quantum Field Theory and Critical Phenomena (Clarendon Press, Oxford, fourth edition, 2001).

24 S. Caracciolo and A. Pelissetto, Nucl. Phys. B 455, 619 (1995).

25 B. Allès, S. Caracciolo, A. Pelissetto, and M. Pepe, Nucl. Phys. B 562, 581 (1999).

26 M. Campostrini, A. Pelissetto, P. Rossi, and E. Vicari, Phys. Lett. B 402, 141 (1997).

27 S. Caracciolo, R. G. Edwards, A. Pelissetto, and A. D. Sokal, Phys. Rev. Lett. 75, 1891 (1995).

28 S. Caracciolo, R. G. Edwards, T. Mendes, A. Pelissetto, and A. D. Sokal, Nucl. Phys. B (Proc. Suppl.) 47, 763 (1996) [hep-lat/9509033].

29 S. Caracciolo, A. Montanari, and A. Pelissetto, Phys. Lett. B 513, 223 (2001).

30 S. Caracciolo and A. Pelissetto, Nucl. Phys. B 420, 141 (1994).

31 Explicitly, we can take $P_{l l} \sim\left(\Phi^{2}\right)^{l / 2} P_{l}(x)$, where $x^{2}=\varphi^{2} / \Phi^{2}$ and $P_{l}(x)$ is a Legendre polynomial.

32 J. Carmona, A. Pelissetto, and E. Vicari, Phys. Rev. B 61, 15136 (2000).

33 E. Brézin, J. Zinn-Justin, and J.C. Le Guillou, Phys. Rev. B 14, 4976 (1976).

34 R.A. Pelcovits and D.R. Nelson, Phys. Lett. A 57, 23 (1976); D.R. Nelson and R.A. Pelcovits, Phys. Rev. B 16, 2191 (1977).

35 K. W. Blazey, H. Rohrer, and R. Webster, Phys. Rev. B 4, 2287 (1971).

36 A. Aharony, Phys. Rev. Lett. 88, 059703 (2002); A. Aharony, J. Stat. Phys. 110, 659 (2003).

37 E. Vicari and J. Zinn-Justin, New Journal of Phys. 8, 321 (2006).

38 R. Leidl and W. Selke, Phys. Rev. B 70, 174425 (2004). 